\title{
An Approach to Embed Image in Video as Digital Watermark
}

\author{
Nagajyothi. T \\ Electronics and Communications, \\ Shri Venkateshwara University, \\ Gajraula, UP, India
}

\author{
Dr. Amit kr.Jain \\ Electronics and Communications, \\ Shri Venkateshwara University, \\ Gajraula, UP, India
}

\begin{abstract}
Digital watermarking covers the secret or personal data in digital images to guard their copyrights. Currently, copyright protection of digital contents, lack of authentication and ownership proof has become a critical issue due to internet growth. To overcome these issues a blind invisible dual video watermarking approach is proposed in this work. Both second level and third level DWT(Discrete Wavelet Transform) are processed in this work. By second level a watermark image (one image embedded over another image) is acquired. The watermark image is then embedded into the third level DWT to provide high level watermarked video. This embedding process is done with only the luminance component of video. Since invisible watermarking approach is utilized the watermark image is given in an invisible form. Consequently, after embedding process extraction process takes place. Thus the watermark image is sent in a secure manner preventing unauthorized usage and copyright issues. The execution time of embedding process and extraction process, Peak Signal to Noise Ratio (PSNR) and Mean Square Error (MSE) and Normalized Correlation Coefficient (NCC) is inspected.
\end{abstract}

Keywords: Blind invisible dual watermarking, digital images, digital watermarking, Mean Square Error (MSE), Normalized Correlation Coefficient and Peak Signal to Noise Ratio (PSNR).

\section{INTRODUCTION}

In ancient times, with the vast improvement of multimedium method, inhabitants could conveniently process or copy variety digital information. All together, the augmentation uses of systematic procedure for multimedium data similar to images, video and audio, the message is released more easily as transmits, also causes the greatest challenge on intellectual property rights, or copyrights protecting [1]. However, the supreme consequence in broadcast of digital data is its simple vulnerability to have inestimable copies of the comparable scenery and eminence as that of the original. So, there is always the chance of the lack of authentication, ownership proof and copyright protection

Digital watermarking is a technique that provides solution for protecting the multimedia file from these activities [2]. A message such as image, audio, video or text can be inserted within the digital media so it should not cause violation to the original digital media [3].

Image watermarking techniques anticipated to this point can be alienated into two groups bestowing to the processing domain of the host image that the watermark is embedded. One is to modify the intensity value of the luminance in the spatial domain [4] which has been shown to be computationally expensive. A watermarking method for color imagery has been anticipated in the wavelet domain in where a visual masking has been enforced on the luminance channel by neglecting the chrominance components. A varying color image watermarking DWTSVD (Singular Value Decomposition) and DCT (Discrete Cosine Transform) with Arnold Cat Map encryption based robust and the blind watermarking scheme is proposed for copyright protection has been proposed in [5].

The embedded watermark is measured as a secreted unfamiliar message that desires to be decoded precisely. It should be noted that the role of a watermark detector is to verify the existence or absence of a hidden message in the original multimedia signal. In fact, it facilitates certifying the ownership and preventing any unauthorized duplication or distribution of digital data. Hence, several image watermarking schemes in the sparse domain have paid attention on conniving watermark detectors by establishing a legitimate decision rule [6]. To determine these concerns, watermarking technique is performed effectual resources for digital invention copyright security [7].

Image watermarking techniques discussed earlier is divided into two groups conceding to the processing domain of host image where the watermark is embedded. Also the watermark has to be embedded in a cherished mode into the host data and while creating the affirmation of the digital data that is extracted effectively [8]. The paper outline is ordered as follows: the recent research work on digital watermarking is described in section 2; section 3 describes the proposed blind invisible dual watermarking approach. Section 4 depicts the simulation results for the work and section 5 ends the work followed by the references.

\section{RELATED RESEARCH}

Some of the recent research techniques related to watermarking techniques and their issues are discussed below:

Yu, Mei et al. [9] Addressed image authenticity confirmation and integrity security concerns. A watermarking scheme for stereo image authentication was proposed with the stereo matching method. Right image blocks and non-similar blocks of left image were classified into soft, surface and composite blocks making an allowance for their coarseness. The alterable-length watermark was figured rooted in the outcome of block classification, and the shattered blocks were recovered by alterable-length watermark. Further, similar damaged 
blocks of left image were improved with the harmonized points in the right image. A detection method with the division of different-measure watermark was utilized to enhance accuracy. The disparity was utilized to recuperate the damaged similar block.

Jagadeesh, B., P. Rajesh Kumar et al. [10] Presented an image watermarking method that exploited the human visual system (HVS), two artificial intelligence techniques [fuzzy inference system (FIS) and back propagation neural networks (BPNN)], and DCT. The incorporation of FIS and BPNN resulted in a hybrid intelligent method called Neuro-fuzzy system. This system used HVS model for conniving the balancing thing by bequeathing the HVS parameters as inputs to the FIS and BPNN. The enumerated weighing value was used for embedding the watermark with utmost power and imperceptibility.

Liu, Shuai et al. [11] depicted the issues with digital goods infringement and patent disagreement that become serious. Here, the authors' developed a digital watermarking scheme derived from a fractal encoding method and DCT. The proposed method combined fractal encoding and DCT for double encryptions to develop a conventional DCT process. The image was encoded by fractal encoding as the first encryption, and then encoded parameters were utilized in DCT method as the second encryption. First, the fractal encoding scheme was accustomed to encode a private image. Then, digital watermarking was summoned to the original image to contradiction utilizing DCT, by which the private image can be extracted from the carrier image with private encoding scales.

Mardolkar, Sukanti B et al. [12] proposed, a blind digital image watermarking algorithm derived from combined DWT-DCT transformation with low frequency watermarking. The watermark embedding was carried out by modifying the wavelet coefficients selected DWT subband of a host image after two levels of DWT decomposition, followed by block based DCT transform on the preferred sub-band. Watermark embedding was done using low frequency coefficients of every DCT block. The watermarked host image was exposed to several watermark attacks.

Malik et al. [13] described a watermarking technique where the watermark image and the extracted watermark images were utilized as the foundation alongside diverse quality parameters to ensure if the imperceptibility of the watermark is retained after watermark extraction. The proposed watermarking scheme is invisible beside diverse quality parameters like PSNR, MSE, maximum difference, and normalized absolute error.

Ozyurt et al [14] demonstrated one layer for watermark embedding. Modulo grounded data hiding process was utilized for watermark embedding. To provide confidentiality of the proposed method, chaotic, random number generator with logistic map was applied. The embedding layer and watermark generation function were selected by using logistic map with modulo operator. The watermark generation step was repeated for tamper detection.

\section{PROPOSED BLIND INVISIBLE DUAL WATERMARKING APPROACH}

In recent times, a prominent technique, digital watermarking, is applied. It identifies applications in the content protection, copyright administration and tamper detection. Content authentication throughout encryption and resistance has turned into an important issue. Imperceptible watermarks hinder illegitimate replication by establishing ownership information unnoticeable embedded into the digital data. Taking into account the authentication of images and copyright protection issues watermarking technique is applied. To determine these issues, a blind invisible dual watermarking approach is proposed in images and video. The steps used in the proposed research are discussed below in Fig 1 and 2. The execution time of watermarking on video is analyzed. NCC is computed for analysis of the proposed blind dual video watermarking technique. Image is embedded in the video and is extracted with acceptable PSNR and MSE values.

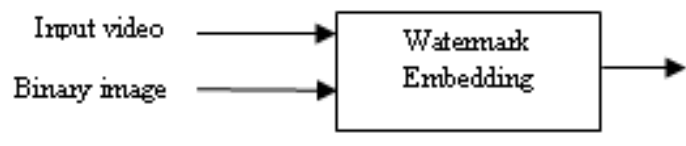

Fig.1. Watermark embedding

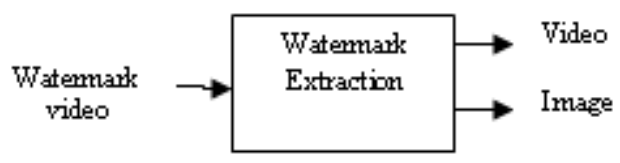

Fig. 2: Watermark Extraction

A blind invisible dual watermarking approach is presented and the main objective is to secure the motion video and simultaneously add the watermark image. Initially a watermark image, also known as a secret image embedded into a video. Generally in most video watermarking technique works with only one image that will be embedded into a video. But in this work rather than a single image, a watermarked image is embedded into the video. By this the watermark image is transmitted in a secure manner, thus preventing unauthorized usage. To obtain a watermark image as in [23], DWT is applied on the region of interest areas of the two given images to get different frequency subbands of its wavelet decomposition generating diverse frequency subbands: LL, HL, LH and $\mathrm{HH}$ as illustrated in figure 3. A pair of elements with similar values is identified from the left singular value matrix from the image. The values of these pairs are modified using certain threshold to embed a bit of watermark content.

By this mechanism two images are made embedded one over the other. This image is termed as watermark image which is invisible or not clear. Consequently a video is given as an input to third level DWT followed by the watermark image and this is embedded in the video. Here to embed the image, the input video is converted to frames. Consequently, in the obtained frames the watermark image is embedded followed by the extraction process. 


\subsection{Watermark Embedding-}

The embedding process of the watermark image into the host video is given in this sub-section. Since blind algorithm is utilized thresholding concept is utilized. The watermark is embedded in the luminance ( $\mathrm{Y}$ ) component of a video frame [18]. The chrominance (U) component guides extra distortion than the $\mathrm{Y}$ component lacking professed by human eyes. Initially the video to be watermarked is divided into different frames. Here the third level DWT technique is utilized for the watermark embedding process. The watermark image is separated into four bands in horizontal direction, vertical direction, diagonal direction and low frequency part that can be decomposed further.

\begin{tabular}{|l|c|c|c|}
\hline LL & LH & \multirow{2}{*}{ LH $^{2}$} & \\
\cline { 1 - 2 } $\mathrm{HL}$ & $\mathrm{HH}$ & & \multirow{2}{*}{ LH' $^{\prime}$} \\
\cline { 1 - 2 } $\mathrm{HL}^{2}$ & & \\
\hline \multicolumn{2}{|c|}{$\mathrm{HL}^{\prime}$} & $\mathrm{HH}^{\prime}$ \\
\hline \begin{tabular}{ll}
\hline 1,2,3-- Deoomposition Levels \\
$\mathrm{H}$ & -- High Frequency Bands \\
L & --- Low Frequency Bands \\
\hline
\end{tabular}
\end{tabular}

Fig.3. Discrete wavelet transform

The three level decomposition of DWT is shown in Fig 3. Initially, the input video is separated into frames by describing the video height and width. Embedding of watermark image into the video frames is done by converting the watermark image to binary pixels by using threshold value. These binary pixel values are stored in an array for future reference i.e. during extraction. Since invisible watermark embedding process, the size $S$ of the original watermark image is obtained. Then the original RGB (Red, Green, and Yellow) video frames is converted into YUV ( $\mathrm{Y}$ is the luminance component, while $\mathrm{U}$ and $\mathrm{V}$ are the chrominance color components) color space. The basic equations used to convert RGB into YUV are as in equation (1), (2) and (3):

$$
\begin{aligned}
& \mathrm{Y}=0.299 \mathrm{R}+0.587 \mathrm{G}+0.114 \mathrm{~B} \\
& \mathrm{U}=-0.14713 \mathrm{R}-0.28886 \mathrm{G}+0.436 \mathrm{~B} \\
& \mathrm{~V}=0.615 \mathrm{R}-0.51499 \mathrm{G}-0 . \quad 10001 \mathrm{~B}
\end{aligned}
$$

The embedding process is done in the $\mathrm{Y}$ frame only. The Y image component is separated into an identical count of $8 \times 8$ blocks and DWT is done on each block by pseudo- random selection. The embedding is done with the video frame, watermark image along with an embedding strength parameter x. Subsequently, DWT performs level by level decomposition with the sub bands (LL, LH, HL, and $\mathrm{HH}$ ). In level 1 decomposition four sub bands LL1, HL1, LH1 and HH1 are obtained, in level 2 four sub bands LL2, HL2, LH2 and HH2 is obtained and finally in level 3 four minor sub bands LL3, HL3, LH3 and HH3 is obtained. The sub bands are concatenated and converted to a decimal value to obtain the size of the watermark image. Consequently resizing of watermark image is done to embed secret mixed image into the video frames. Then the watermark is embedded into the video frames thus obtaining watermarked sub bands. Consequently frame regeneration is done by inverse DWT and produce new sub bands as in algorithm 1. After embedding the corresponding frame is changed back to RGB using equation (4), (5) and (6):

$$
\begin{aligned}
& \mathrm{R}=\mathrm{Y}+1.13983 \mathrm{~V} \\
& \mathrm{G}=\mathrm{Y}-0.39465 \mathrm{U}-0.58060 \mathrm{~V} \\
& \mathrm{~B}=\mathrm{Y}+2.03211 \mathrm{U}
\end{aligned}
$$

Once the embedded frame is obtained watermark extraction is done followed by concatenation of the YUV color spaces to obtain the color image. The flowchart in Fig 4 and algorithm 1 explains the watermark embedding process.

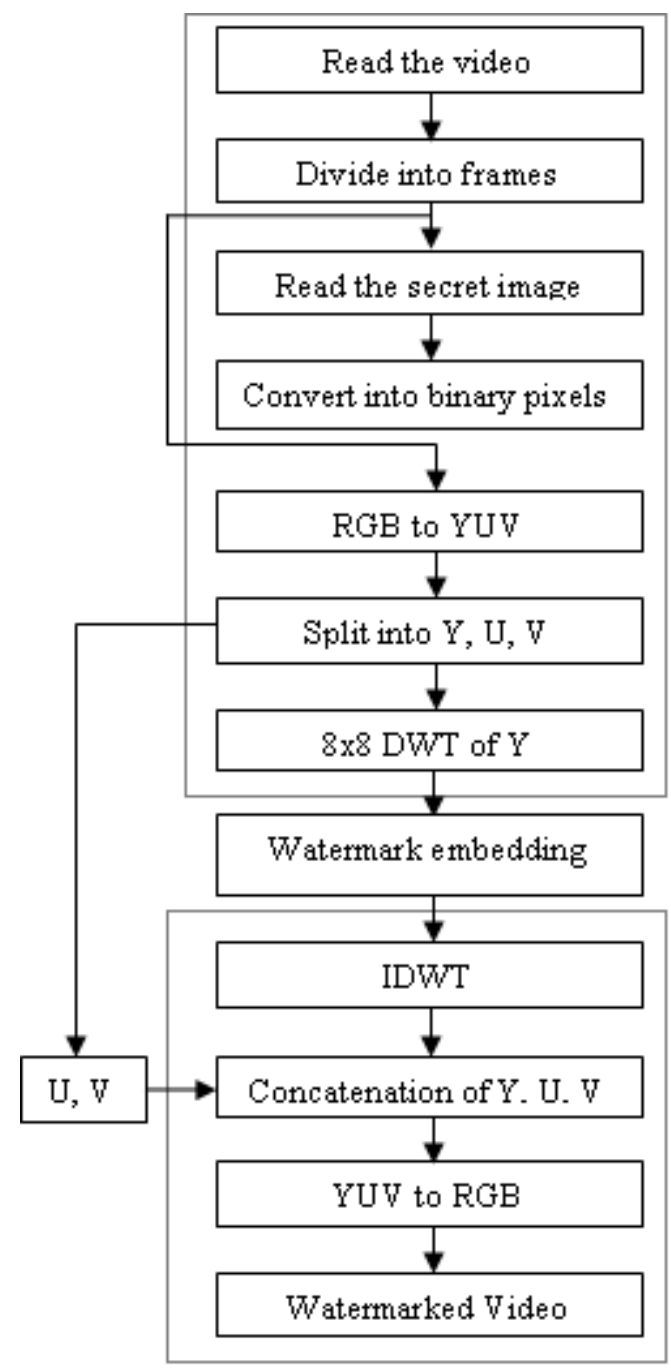

Fig. 4. Flowchart for watermark embedding process

$$
\begin{aligned}
& \text { Algorithm 1: Watermark embedding process } \\
& \text { Input } \quad \text { : video, watermark image } \\
& \text { Output } \quad \text { : embedded image }
\end{aligned}
$$

1. The input video is decomposed into different frames

2. Perform first level DWT on frames to decay, it into four sub bands LL1, HL1, LH1 and HH1. 
3. Then second level DWT is achieved on the LL1 sub band to get four sub bands LL2, HL2, LH2 and $\mathrm{HH} 2$.

4. Consequently, third level DWT is achieved on the LL2 sub band to get four minor sub bands LL3, HL3, LH3 and HH3.

5. First level DWT executed on the watermark image decay, it into four sub bands wLL1, wHL1, wLH1 and $\mathrm{wHH} 1$.

6. Then second level DWT is executed on the LL1 sub band to obtain four sub bands wLL2, wHL2, wLH2 and $\mathrm{wHH} 2$.

7. The third level DWT is executed on the LL2 sub band and four sub bands w LL3, wHL3, w LH3 and wHH3 is obtained.

8. An embedding function ' $a$ ' is used to add the two sub bands as in equation (7):

$$
\text { New LL3 }=\text { LL3 + a* wLL3 }
$$

9. Now Inverse DWT (IDWT) is executed utilizing the sub bands LL3, LH3, HL3, HH3 to acquire image new LL2.

10. Also IDWT is achieved using the sub bands newLL2, LH2, HL2, HH2 to get image new LL1.

11. Finally IDWT is performed with newLL2, LH1, HL1, and HH1 to get the watermarked image.

12. This watermarked image is embedded in the input video frames.

\subsection{Watermark Extraction Process-}

The watermark extraction is the reverse of the watermark embedding procedure. The watermark embedded video frame from the watermark embedding process is given as input to the watermark extraction process. As in embedding the embedded RGB frame is changed into YUV color space. The watermark extraction is done in $\mathrm{Y}$ frame only since embedding is also performed only with the $\mathrm{Y}$ frame. The watermark extraction process is done with the watermarked frame, original video frame, embedding strength parameter $\mathrm{x}$, and size of the watermark image.

The DWT performs three levels of decomposition with the watermarked frames to produce sub bands. In level 1 the sub bands obtained include fLL1, fHL1, fLH1 and fHH1, as that of level 2 include fLL2, fHL2, fLH2 and fHH2 and finally fLL3, fHL3, fLH3 and fHH3 in level 3. The sub bands are concatenated and converted to a decimal value to obtain the size of the watermark image. Consequently resizing of watermark image is done to extract it from video frames. Then the watermark is extracted into the video frames thus obtaining watermarked sub bands. Consequently frame regeneration is done by IDWT and produce new sub bands as in algorithm 2. This process is repeated for up to eight times to get the extracted watermark. The existence of watermark is checked by the condition $x>$ th where th is the threshold. If the condition is satisfied, then watermark is present and if not the watermark is absent. After embedding the corresponding frame is changed back to RGB color frame and is illuminated in Fig 5.
Algorithm 2: Watermark extraction process

Inputs: watermark embedded video

Output: extracted watermark, video

1. First level DWT is performed on the host frame to decay, it into four sub bands fLL1, fHL1, fLH1 and fHH1.

2. Then second level DWT is executed on the LL1 sub band to acquire four sub bands fLL2, fHL2, fLH2 and $\mathrm{fHH} 2$.

3. The third level DWT is executed on the LL2 sub band to acquire four sub bands fLL3, fHL3, fLH3 and fHH3.

4. First level DWT is executed on the watermarked image to decompose it into four sub bands nLL1, nHL1, nLH1 and nHH1.

5. The second level DWT is executed on the LL1 sub band to acquire four sub bands nLL2, nHL2, nLH2 and nHH2.

6. The third level DWT is executed on the LL2 sub band to acquire four sub bands n LL3, n HL3, n LH3 and nHH3.

7. Extraction is done to acquire wLL3 with the same value of ' $a$ ' as in embedding as in equation (8):

$$
\text { wLL3 = newLL3 - LL3 / a }
$$

8. Perform inverse DWT on wLL3 with all other sub bands (LH, HL, HH) equal to 0 to acquire wLL2

9. Step 8 is done over two times all levels to obtain the extracted watermark.

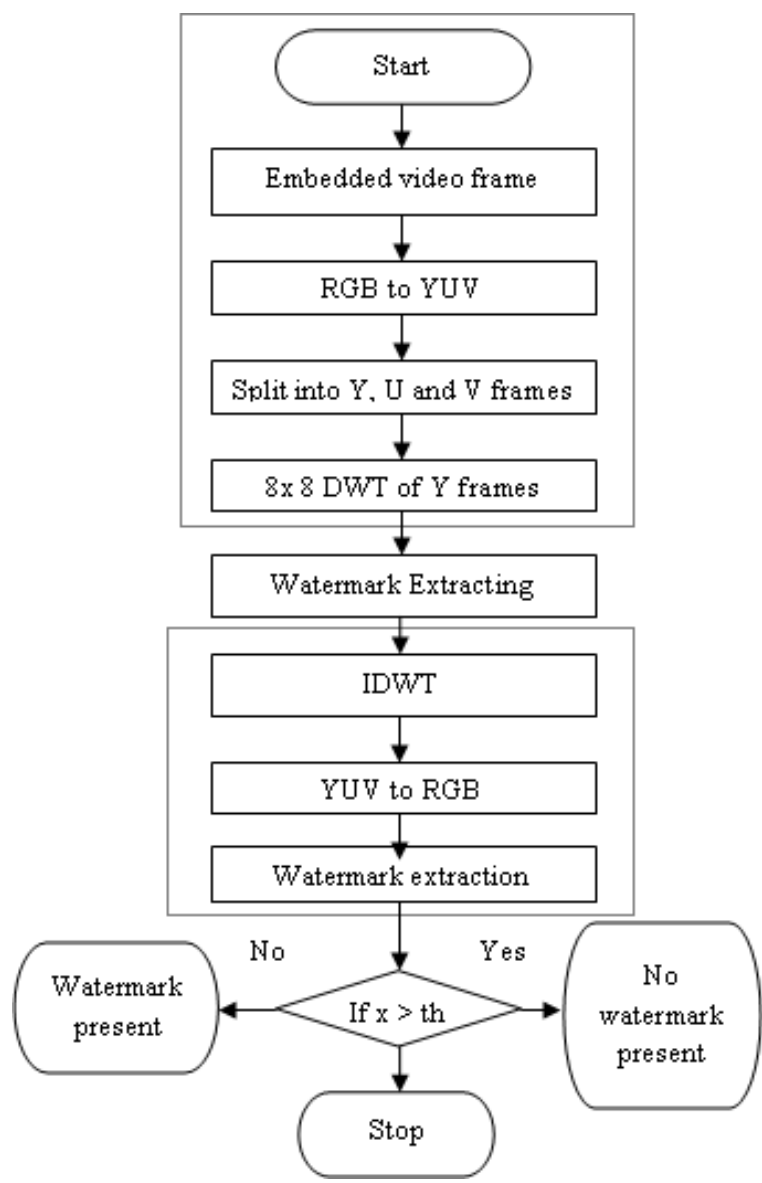

Fig.5. Flowchart for watermark extraction process 


\section{PERFORMANCE ANALYSIS}

The main objective of the work is to resolve the copyright protection without any loss in image quality. The experimental results of the proposed blind invisible dual watermarking approach are presented in this section. The proposed watermarking approach is programmed in Matlab and tested with video (.mpg file of size $352 \times 288$ with 210 frames) and watermark image obtained by second level DWT is resized to $198 \times 256$ of .png file format. The two images utilized to obtain a watermark image are of dimensions 2048 × 1024 and 345 x 206 respectively. The threshold value utilized during watermarking two images is 70. The size of the watermark image is reshaped during embedding and extracting to match with the size of the video.

The watermark image is embedded into the video effectively. Subsequently, the embedded watermark extracts from the watermarked video efficiently. The blind dual technique is achieved by utilization of an invisible watermark image with visible video. From the results it is viewed that the visual quality of the image is preserved so that the alteration in the image quality before and after watermarking cannot be professed by human eye. Here PSNR and MSE are determined as the factors to determine image quality. The watermarked images have good PSNR, MSE is supposed to have good visual quality. Another advantage is that only the luminance part (Y) of the image is considered this improves the computational efficiency as only one color component has to be processed. Other than this normalized correlation coefficient (NCC) parameter is given to measure the quality of extracting watermarks.

\subsection{Simulation Results-}

The simulation result of the proposed blind dual invisible watermarking approach is presented in this section. The watermark embedding strength is defined hence degradation in watermarked image is invisible and distortion in extracting watermark is minimized (i.e. PSNR and NCC of extracting watermark are maximized). Small values of embedding strength parameter can formulate the watermark weak to alteration and also hard to extract and detect during the detection/extraction stage. Similarly, large values of embedding strength parameter can make the watermark visible. This watermark embedding strength parameter dictates the perceived quality and strength of the watermark in the watermarked video frames [20]. In this work the value of watermark embedding strength is assumed to be $0.1,3.1,6.1$ and 9.1 and also can be assumed from 0.1 to 10 .

\subsubsection{Execution Time-}

Execution time is defined as the time taken to construct a watermark (comprising of both extraction time and embedding time) i.e. time taken to complete the execution. The time taken to load video frames are also analyzed for different embedding strengths like 0.1, 3.1, 6.1, 9.1 are tabulated in a table 1 .
Table 1: Table describing embed time, extract time and video load time

\begin{tabular}{|l|l|l|l|}
\hline $\begin{array}{l}\text { Embedding } \\
\text { Strength (x) }\end{array}$ & $\begin{array}{l}\text { Video load } \\
\text { time (sec) }\end{array}$ & $\begin{array}{l}\text { Embed } \\
\text { time(sec) }\end{array}$ & $\begin{array}{l}\text { Extract time } \\
\text { (sec) }\end{array}$ \\
\hline 0.1 & 3.252782 & 32.25687 & 9.569985 \\
\hline 3.1 & 21.824276 & 41.11630 & 52.113621 \\
\hline 6.1 & 62.910744 & 50.755888 & 20.846105 \\
\hline 9.1 & 77.141804 & 39.052694 & 16.438736 \\
\hline
\end{tabular}

Table 1 clears the varying time for varying embedding strength values.

\subsubsection{Mean Square Error (MSE)-}

The MSE value of the proposed blind invisible dual watermarking approach varies for every embedding strength and is tabulated in table 2. This parameter compares the extracted watermark to that of the original video. The MSE value is thus calculated by using the formula in equation (9):

$$
M S E=\frac{\sum_{M, N}\left[I_{1}(m, n)-I_{2}(m, n)\right]^{2}}{M * N}
$$

Where $\mathrm{M}$ and $\mathrm{N}$ is the number of rows and columns in watermarked invisible image.

\subsubsection{Peak Signal and Noise Ratio (PSNR)-}

The PSNR value for the proposed blind invisible dual watermarking approach also varies in different embedding strength as tabulated in table 2. The PSNR value is estimated with respect to MSE. The PSNR is measured between the host video and the watermarked image is and this measure the invisibility of the watermark. The PSNR value is calculated by using the formula in equation (10):

$$
P S N R=10 \log _{10}\left(\frac{255 * 255}{M S E}\right)
$$

The value of the PSNR determines the visibility of the extracted watermark image [16]. Thus, proposed blind invisible dual watermarking approach has better watermark invisibility with a high PSNR value Figure 9 illustrates the extracted watermark which is clearly invisible. Also the original video, embedded video and the watermark image are depicted in Figure 6,7 and 8 respectively.

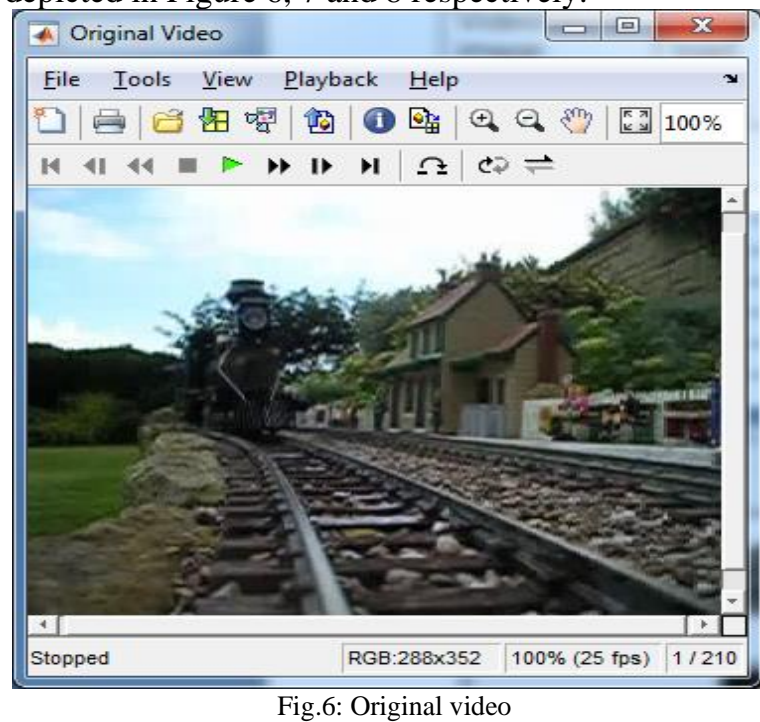




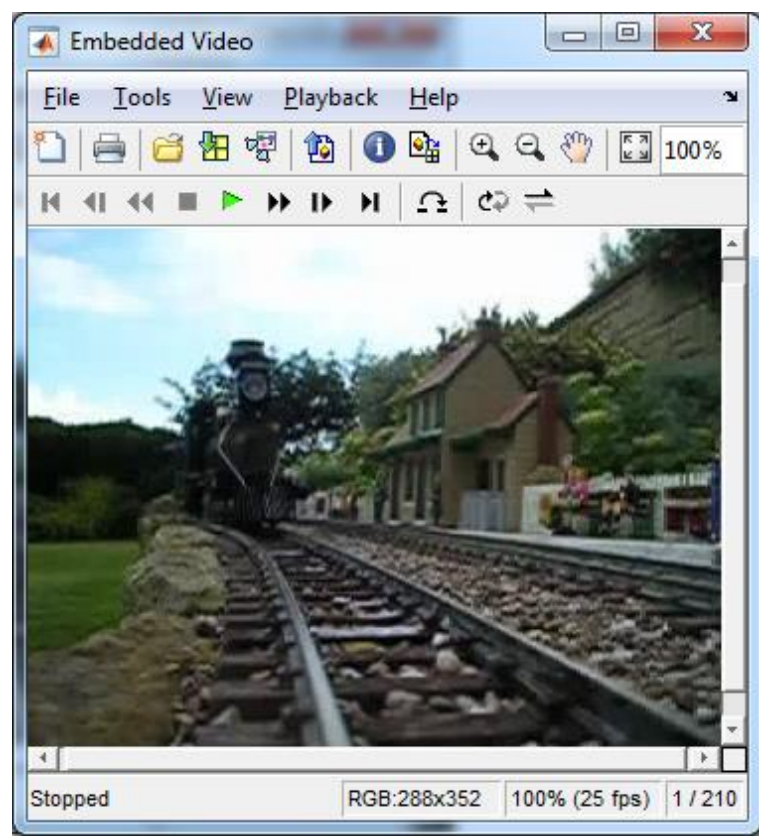

Fig.7: Embedded video

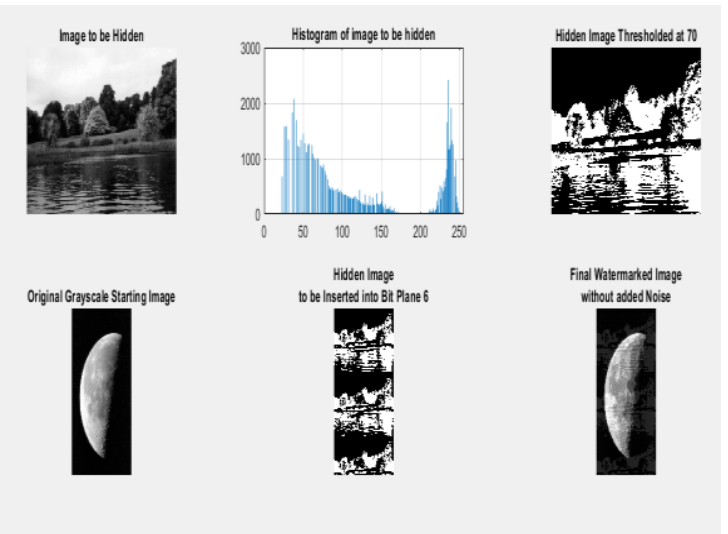

Fig.8. Watermark image

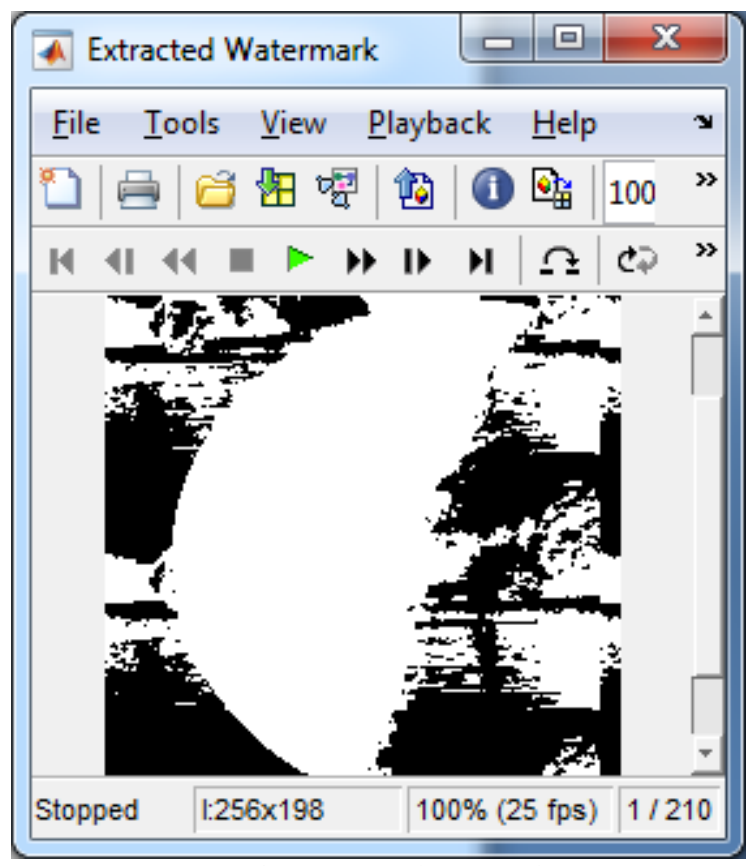

Fig.9. Extracted watermark

\subsubsection{Normalized Correlation Coefficient (NCC)}

To evaluate the quality of an extracted watermark, the similarity between the original video to be watermarked and the extracted video is calculated as the NCC. The NCC can be given as follows as in equation (11):

$$
\mathrm{NCC}=\frac{\text { originalwatermarkimage* extractedwatermark }}{\sqrt{(\text { originalwatermarkimage })^{2}} \sqrt{(\text { extractedwatermark })^{2}}}
$$

The NCC for the proposed approach is 1 for all embedding strength and is depicted in table 2 .

Table 2: NCC, PSNR, MSE values for different embedding strength

\begin{tabular}{|l|l|l|l|}
\hline $\begin{array}{l}\text { Embedding } \\
\text { strength }\end{array}$ & MSE & PSNR & NCC \\
\hline 0.1 & 0.0068 & 160.7011 & 1 \\
\hline 3.1 & 0.2115 & 126.3612 & 1 \\
\hline 4.1 & 0.4161 & 119.5924 & 1 \\
\hline 9.1 & 0.6208 & 115.5925 & 1 \\
\hline
\end{tabular}

Thus, from table 2 it is evident that all the values of PSNR and MSE varies indirectly proportional in different embedding strength. Though NCC is identified constant for all varying embedding values. For comparison with other techniques the values of 0.1 embedding strength are is utilized.

\subsection{Comparison Results-}

Table 3 describes the comparison results between the proposed blind invisible dual watermarking approaches with some other existing approaches.

\begin{tabular}{|l|l|l|l|l|}
\hline \multicolumn{5}{|c|}{ Table -3 Comparison Results } \\
\hline $\begin{array}{l}\text { Proposed } \\
\text { approach }\end{array}$ & 160.7011 & 0.0068 & $\begin{array}{l}\text { Blind dual } \\
\text { invisible }\end{array}$ & 1 \\
\hline $\begin{array}{l}{[17] \text { Blind dual }} \\
\text { watermarking }\end{array}$ & 40.32 & 1.1756 & Blind dual & 0.9894 \\
\hline $\begin{array}{l}{[\mathbf{1 9 ]} \text { Watermark }} \\
\text { embeds and } \\
\text { blind extraction } \\
\text { algorithm }\end{array}$ & 44.78 & 1.21244 & $\begin{array}{l}\text { Blind } \\
\text { invisible }\end{array}$ & 0.89 \\
\hline $\begin{array}{l}{[21] \text { Paillier }} \\
\text { homomorphic } \\
\text { cryptosystem } \\
\text { with poker } \\
\text { shuffling } \\
\text { transformation- } \\
\text { based } \\
\text { watermarking } \\
\text { method }\end{array}$ & 56.4919 & 0.2831 & - & 0.999 \\
\hline $\begin{array}{l}{[22] \text { Robust }} \\
\text { blind and secure } \\
\text { watermarking }\end{array}$ & 45.07 & 1.1434 & Blind & 1 \\
\hline
\end{tabular}




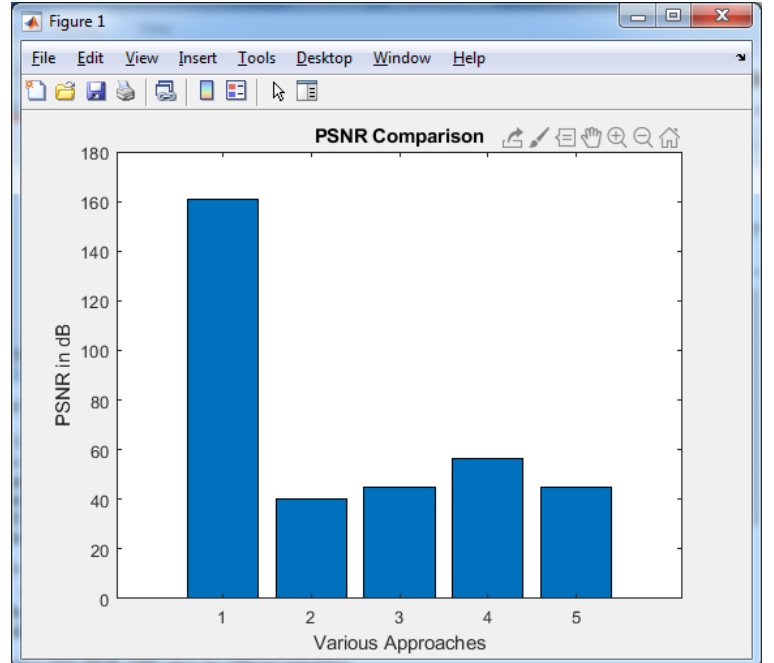

Fig.10. PSNR comparison graph

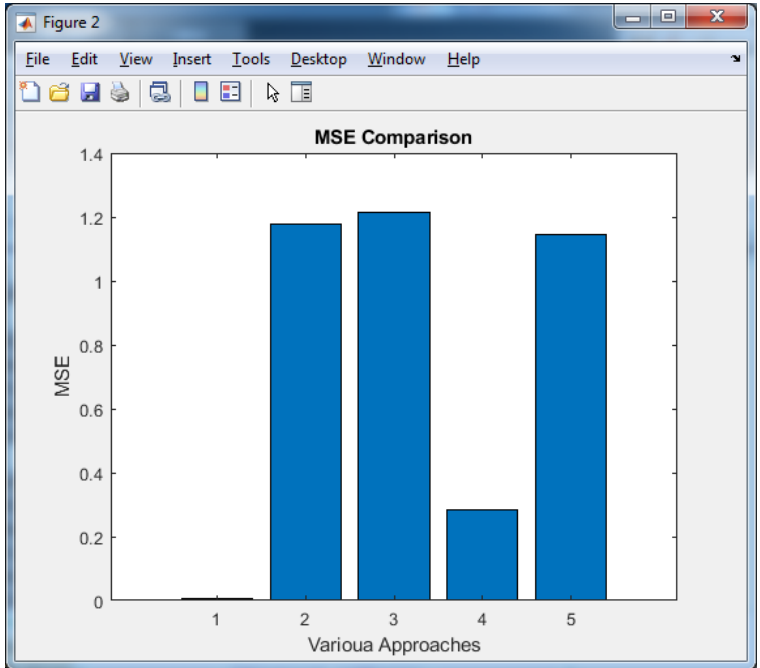

Fig.11. MSE comparison graph

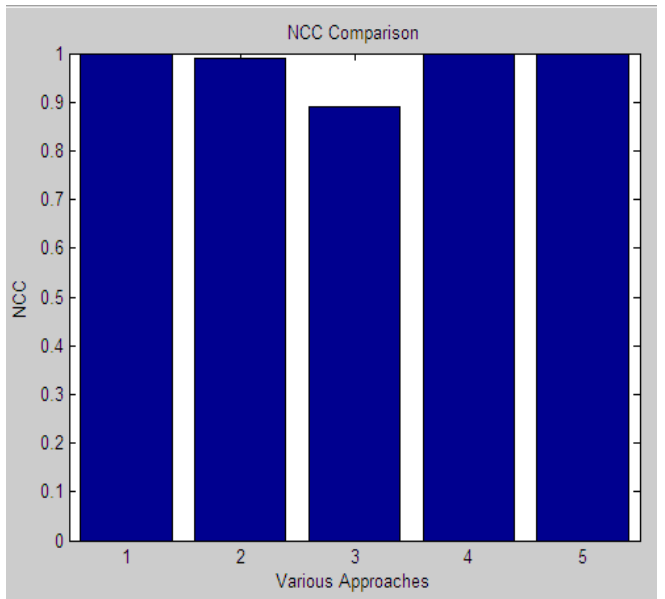

Figure12. NCC comparison graph

Table 3 lists the MSE, PSNR, and NCC of the proposed watermarking approach. It can be experimented that the proposed scheme has higher PSNR value when compared to other methods in reference. As the visual quality of the watermarked images enhances with larger values of PSNR, this outcome depicts that the proposed approach manages the quality of the watermarked images so that it is unfeasible for the human eye to visually perceive the existence of any watermark in it. Higher values of PSNR also show how strong the approach is to diverse types of attack. This is illustrated in Fig 10. The higher value of MSE for the proposed approach with the existing approaches determines the improved quality of the watermark. This explanation can be viewed pictorially in Fig 11. The high NCC values in Table 1 when compared with existing watermarking approaches portray that the scheme is free from attacks. Additionally, it proves that the quality of the extracted watermark is preserved. This higher value of NCC is obtained due to selection of pseudo random generation which selects collections of embedding square luminance blocks for the successive frames of the video. The comparison graph for NCC graph is portrayed in Fig 12.

\section{CONCLUSION}

Digital watermarking is the method to embed the secret data within text, Image or video for ownership verification. Here, a blind invisible dual watermarking mechanism is presented for authentication of images and copyright protection. The invisible watermarking used maintains the ownership needs since the extracted watermark is not visible through human eyes. Comparisons of the proposed approach with the performances of other watermarking mechanisms clearly demonstrated in table 3 depict the superiority of the proposed approach. The performance analysis is given with different metrics like PSNR to measure the invisibility of the approach and is viewed as 160.7011dB. The other metrics such as MSE and NCC are meant to depict the quality of the watermark by similarity measures. The value for MSE for proposed approach is 0.0068 and that of the NCC is 1 which proves the watermark is free from attacks. Further research will be done to make the algorithm work if the watermark embedding and extraction process is to be done to maximize the security with different attacks.

\section{ACKNOWLEDGEMENT}

The successful completion of any task would be incomplete without mentioning the people who made it possible through their constant guidance and encouragement. I (Nagajyothi T) express my gratitude to Dr.Amit Jain, Ph.D my guide for his inspiration and guidance throughout my study. The interest he showed in my project was admirable and his encouragement motivated me to give my best.

I take this opportunity to express my sincere thanks to Dr. Vipin Kumar, Dy.Registrar (Research \& Planning) for his constant encouragement throughout the study.

\section{REFERENCES}

[1]. Singh, Amit Kumar. "Improved hybrid algorithm for robust and imperceptible multiple watermarking using digital images." Multimedia Tools and Applications Vol.76, no. 6, pp. 8881-8900, 2017.

[2]. Rangel-Espinoza, Kevin, Eduardo Fragoso-Navarro, Clara CruzRamos, Rogelio Reyes-Reyes, Mariko Nakano-Miyatake, and Héctor M. Pérez-Meana. "Adaptive removable visible 
watermarking technique using dual watermarking for digital color images." Multimedia Tools and Applications Vol. 77, no. 11, pp. 13047-13074, 2018

[3]. Priya, S., R. Varatharajan, Gunasekaran Manogaran, Revathi Sundarasekar, and Priyan Malarvizhi Kumar, "Paillier homomorphic cryptosystem with poker shuffling transformation based water marking method for the secured transmission of digital medical images", Personal and Ubiquitous Computing, pp. 1-11, 2018

[4]. Sun, Guangmin, Rui Wang, Shu Wang, Xiaomeng Wang, Dequn Zhao, and Andi Zhang, "High-definition digital color image watermark algorithm based on QR code and DWT", In Industrial Electronics and Applications (ICIEA), IEEE 10th Conference, pp. 220-223, 2015.

[5]. Singh, Durgesh, and Sanjay K. Singh, "DWT-SVD and DCT based robust and blind watermarking scheme for copyright protection", Multimedia Tools and Applications, Vol. 76, No. 11, pp. 1300113024, 2017.

[6]. Amini, Marzieh, Hamidreza Sadreazami, M. Omair Ahmad, and M. N. S. Swamy, "A channel-dependent statistical watermark detector for color images", IEEE Transactions on Multimedia, Vol. 21, No. 1, pp. 65-73, 2019.

[7]. Ambadekar, Sarita P., Jayshree Jain, and JayshreeKhanapuri, "Digital Image Watermarking Through Encryption and DWT for Copyright Protection", Recent Trends in Signal and Image Processing, Springer, pp. 187-195, 2019.

[8]. Makbol, Nasrin M., Bee Ee Khoo, Taha H. Rassem, and Khaled Loukhaoukha. "A new reliable optimized image watermarking scheme based on the integer wavelet transform and singular value decomposition for copyright protection." Information Sciences Vol. 417, pp. 381-400, 2017.

[9]. Yu, Mei, Jing Wang, Gangyi Jiang, Zongju Peng, Feng Shao, and Ting Luo, "New fragile watermarking method for stereo image authentication with localization and recovery", AEU-International Journal of Electronics and Communications, Vol. 69, No. 1, 361370, 2015.

[10]. Jagadeesh, B., P. Rajesh Kumar, and P. Chenna Reddy, "Robust digital image watermarking based on fuzzy inference system and back propagation neural networks using DCT", Soft Computing, Vol. 20, No. 9, pp. 3679-3686, 2016.

[11]. Liu, Shuai, Zheng Pan, and Houbing Song, "Digital image watermarking method based on DCT and fractal encoding", IET image Processing, Vol. 11, No. 10, pp. 815-821, 2017.

[12]. Mardolkar, Sukanti B., and Nayana Shenvi, "A Blind digital Watermarking Algorithm based on DWT-DCT Transformation", International Journal of Innovative Research in Electrical, Electronics, Instrumentation and Control Engineering, Vol. 3, No. $2,2016$.
[13]. Dziech, Andrzej, and Jakob Wassermann, "Application of Enhanced Hadamard Error Correcting Code in VideoWatermarking and his comparison to Reed-Solomon Code", MATEC Web of Conferences, 125, 2017.

[14]. Malik, Gunjan, Tarun Kumar, and Gaurav Agarwal, "Wavelet Analysis in Video Watermarking through Wavelet Transform Scheme “, International Journal of Computer Applications, Vol. 161, No. 4, 2017.

[15]. Ozyurt, Fatih, TurkerTuncer, and EnginAvci, "A Novel Probabilistic Image Authentication Method based on Universal Hash Function for Rgb Images", Computing Sciences and Engineering (ICCSE), International Conference on. IEEE, pp. 1-6, 2018.

[16]. Imran, Muhammad, Bruce A. Harvey, and Adnan Ali Memon. "A novel blind color image watermarking technique based on singular value decomposition and principal component analysis." In Sixth International Conference on Innovative Computing Technology (INTECH), pp. 519-524. IEEE, 2016.

[17]. Liu, Xiao-Long, Chia-Chen Lin, and Shyan-Ming Yuan, "Blind dual watermarking for color images' authentication and copyrigh protection", IEEE Transactions on Circuits and Systems for Video Technology, Vol. 8, No. 5, pp. 1047-1055, 2018

[18]. Ingale, Sumedh P., and C. A. Dhote, "Digital Watermarking Algorithm using DWT Technique", International Journal of Computer Science and Mobile Computing, Vol. 5, No. 5, pp. 0109,2016

[19]. Albalawi, Umar, Saraju P. Mohanty, and Elias Kougianos, "A new region aware invisible robust blind watermarking approach", Multimedia Tools and Applications, Vol. 76, No. 20, pp. 21303 21337, 2017.

[20]. Ahuja, Rakesh. "Design of Digital Video Watermarking Technique Based on Motion Frames." Journal of Computational and Theoretical Nanoscience, Vol. 16, no. 10, pp. 4328-4338, 2019.

[21]. Priya, S., R. Varatharajan, Gunasekaran Manogaran, Revathi Sundarasekar, and Priyan Malarvizhi Kumar, "Paillie homomorphic cryptosystem with poker shuffling transformation based water marking method for the secured transmission of digita medical images", Personal and Ubiquitous Computing, pp. 1-11, 2018.

[22]. Farri, Elhameh, and Peyman Ayubi, "A blind and robust video watermarking based on IWT and new 3D generalized chaotic sine map", Nonlinear Dynamics, pp. 1-23, 2018.

[23]. Thakkar, Falgun N., and Vinay Kumar Srivastava. "A blind medical image watermarking: DWT-SVD based robust and secure approach for telemedicine applications." Multimedia Tools and Applications Vol. 76, no. 3, pp. 3669-3697, 2017. 